International Journal of Agriculture, Environment and Bioresearch

Vol. 06, No. 04; 2021

ISSN: $2456-8643$

\title{
DETERMINATION OF EFFICIENT HERBICIDE IN SUPPRESSING WEED SPECIES, FAMILY FOR BETTER CROP GROWTH AND YIELD OF BORO RICE CULTIVATION IN BANGLADESH
}

\author{
Md. Shahidul Islam ${ }^{1}$ and Md. Ibrahim Ali ${ }^{1}$ \\ ${ }^{1}$ Principal Scientific Officer, Agronomy Division, Bangladesh Institute of Nuclear Agriculture, Mymensingh-2202, \\ Bangladesh. \\ ${ }^{1}$ Scientific officer, Agronomy Division, Bangladesh Institute of Nuclear Agriculture, Mymensingh-2202, \\ Bangladesh.
}

https://doi.org/10.35410/IJAEB.2021.5649

\begin{abstract}
A field experiment was conducted Agronomy Divisions field Laboratory of the Bangladesh Institute of Nuclear Agriculture (BINA), Mymensingh under wet land condition during January to May 2019 to determine the efficiency of different classes of herbicide. There is scarcely works on determination of efficiency of available 15 classes of herbicide in Bangladesh. The objectives was to know the efficiency of herbicide in suppressing the flora of weeds species and family, analysis of crop growth parameters and yield of boro rice. The experiments were laid out in a randomized complete block design (RCBD) with three replications and 20 treatments. In this experiment highest weed control efficiency was found at penoxsulam $86.55 \%$ which is followed by (bensulfuron methyl + acetachlor), $84.25 \%$ and lowest glyphosate $54.62 \%$ treated plot. The chemical classes (bensulfuron methyl + acetachlor, pretilachlor, pyrazosulfuron ethyl, ethoxysulfuron, bispyriback sodium, bensulfuron methyl + bispyriback sodium, penoxsulam, pyrazosulfuron ethyl + pretilachlor) WCE \% more than 80 . Seventeen weed species belongs to six families namely Gramineae, Pontederiaceae, Cyperaceae, Asteraceae, Marsileaceae and Sphenocleaceae were found to grow in weedy check plots. The highest summed dominance ration of weed was 10.5 for Cyperus difformis. The infestation of different categories weed maximum relative weed density was observed for Echinochloa colona, Cyperus difformis, Leersia hexandra $L$. The highest grain yield (6.83 t ha-1) was found with penoxsulam treatment which was followed by (6.82 $\mathrm{t}$ ha-1) at Bensulfuran methyl+ Bispyriback sodium treatment and lowest (4.61 t ha-1) at control. This results indicates that boro rice could be grown with uses of efficient herbicides classes (bensulfuron methyl + acetachlor, pretilachlor, pyrazosulfuron ethyl, ethoxysulfuron, bispyriback sodium, bensulfuron methyl + bispyriback sodium, penoxsulam, pyrazosulfuron ethyl + pretilachlor) to maximize yield of boro rice.
\end{abstract}

Keywords: Herbicide, bensulfuron methyl, acetachlor 14\%, 2-4, D-amine, pretilachlor, triafemon, pyrazosulfuron ethyl, trisulfuron, pendimethalin, metsulfuron methyl, butachlor, ethoxysulfuron, carfentrazol ethyl, fenoxpro-p-ethyl, bensulfuron methyl, bispyriback sodium, penoxulam, bispyriback sodium, paraquate dichloride, glyphosate, weed density, weed species. 


\section{International Journal of Agriculture, Environment and Bioresearch}

Vol. 06, No. 04; 2021

ISSN: $2456-8643$

\section{INTRODUCTION}

Rice (Oryza sativa L.) is one of the oldest cultivated cereal crops in the history of mankind (Chauhan, 2013). It is considered a staple food, providing the daily caloric requirements for many people in Latin America, North Africa, Asia, the Caribbean, and sub-Saharan countries (Siwar et al., 2014). Approximately half of the world's population staple food rice (Jabran and Chauhan 2015). Rice producing countries central Africa, Japan, Indonesia, central and South America, Malaysia, the Philippines, India, China, Australia, and Italy (Kaloumenos et al., 2013). Bangladesh is a densely populated country. She has to feed nearly 170 million mouths from an area of 8 million ha of cultivable land. Rising of labor costs in the transplanted seedling approach represent approximately $79 \%$ of the total cost of rice production per hectare (Najim et al., 2007). In Bangladesh increasing labor crisis in the periods of peak demand and high wage of labor encourages farmers to use herbicides for weed control in rice field (Krishna, V. et al., 2012). To keep in good terms of food security, it is quite important productivity levels increasing of rice in adverse impacts of climate change. Weeds management is major yield limiting factors in rice cultivation. It creates negative impact on crop production and responsible for $45-55 \%$ reduction of rice yield (Ghosh et al., 2013) in case of severe infestation. Previous studied revealed that application of herbicides reported to increase 30 to 40 per cent yield over control in transplanted rice (Bari, 2010). There have been tremendous developments in herbicide technology a wide spectrum range of pre-emergence and post-emergence herbicides now available to rice growing farmers. Application increases due to Improvement selectivity and formulations allow safer, easier, cheap and more flexible application for rice crops. The new way of growing rice, weed control relies heavily on herbicides. Selective herbicides kill specific targets, while leaving the non-targeted planted crops. Herbicides are commonly used due to quick, effective and low cost involvement in rice production system throughout the world (Hossain, M. M.et al., 2015). Nowadays use of herbicides is gaining popularity in rice culture due to their rapid effects and less cost involvement. The efficacy of herbicide in controlling weeds is important. After a field survey from dealers and chemical supplying companies (ACI Crop Care, Auto Crop Care, Macdonald Bangladesh, Intefa, Syngenta Bangladesh, Petrochem Bangladesh, Bayer crop science, National Agricare Import and export, SAM, SEMCO, Agriplus) on availability of common categories of chemical herbicides are used by the farmers are bensulfuron methyl, acetachlor, 2-4 d amine, pretilachlor, bispyriback-sodium, pyrazosulfuron ethyl, sulfonanilide, pendimethyline, metsulfuron methyl, butachlor, penoxsulam, pyrazosulfuron ethyl, pretilachlor, ethoxisulfuron, trisulfuron, machete, carfentrazon ethyl, cyalopbutyl, fenoxpro-pre-ethyl, orthosulfomuron, anilphos, cinmethyl, cinosulfuran, butachlor, glyphosate, paraquate, mefnaset, metsulfuron+chloromuron, MCPA remarkably used in our country. Selectivity unnecessarily dependent upon the compounds, but also on the rates, timing and methods of application as important to follow the manufacturers labelled recommendations. Indiscriminate uses and subsequent dispersion of herbicide compounds and their degradation products in rice fields may hampers the ecosystems. Herbicides can be toxic to humans at higher and lower doses (Zeliger et al., 2011). Soil composition also affects herbicide phytotoxicity and persistence through adsorption, leaching, and volatilization. Soils high in clay, organic matter, or both have a greater potential for herbicide carryover because there is increased adsorption to soil colloids, with a corresponding decrease in leaching and volatilization losses. The efficiency, persistence and mobility of herbicides are influenced by agro-climatic conditions. The information on efficiency 
of chemical compounds, dose, time and precaution for uses and predict the behavior of herbicides to suppress particular weed species and families for particular topography and soil types. Rice production systems rely on herbicides and the evolution of new weed problems and herbicide resistant ecotypes. It is time to emphasis on the judicious use of herbicides. From consideration of those facts the experiment was conducted.

\section{MATERIALS AND METHODS}

\subsection{Experimental site}

The experiment was carried out at the Field Laboratory of Agronomy Division, Bangladesh Institute of Nuclear Agriculture, head quarter farm Mymensingh, boro season during January to May 2019. The experimental field was located at $24.75^{\circ} \mathrm{N}$ latitude and $90.50^{\circ} \mathrm{E}$ longitudes having an elevation of $18 \mathrm{~m}$ above from sea level. Initial soil sample was randomly taken and analysis of composite sample to determine the soil morphological, physical and chemical characteristics of the experimental field.

Table 1. Soil morphological, physical and chemical characteristics of the experimental field

\begin{tabular}{|l|l|}
\hline Morphological characteristics & \\
\hline Agro-ecological zone (AEZ) & Old Brahmaputra Floodplain (AEZ 9) \\
\hline General Soil Type & $\begin{array}{l}\text { Non-calcareous Dark Grey Floodplain Soils Parent } \\
\text { material, Brahmaputra river borne deposits }\end{array}$ \\
\hline Topography & Medium high land and moderate drainage capacity \\
\hline Order & Inceptisol \\
\hline Physical Characteristics & 22.7 \\
\hline$\%$ Sand & 64.2 \\
\hline$\%$ Silt & 13.1 \\
\hline \% Clay & Silt loam \\
\hline Textural Class & $($ soil : water=1:2.5) 6.6 \\
\hline Chemical characteristics & 2.69 \\
\hline Interpretation pH & $\left(\mathrm{col} \mathrm{kg}{ }^{-1} 0.09\right)$ \\
\hline Organic matter (\%) & \\
\hline Low Total N & \\
\hline
\end{tabular}


International Journal of Agriculture, Environment and Bioresearch

Vol. 06, No. 04; 2021

ISSN: $2456-8643$

\begin{tabular}{|l|l|}
\hline Low Available $\mathrm{P}$ & $\left(\mathrm{mg} \mathrm{kg}^{-1} 3.00\right)$ \\
\hline Very low Available $\mathrm{S}$ & $\left(\mathrm{mg} \mathrm{kg}^{-1} 14.32\right)$ \\
\hline Low Available $\mathrm{Zn}$ & $\left(\mathrm{mg} \mathrm{kg}^{-1} 0.69\right)$ \\
\hline Low Available B & $\left(\mathrm{mg} \mathrm{kg}^{-1} 0.24\right)$ \\
\hline Low Available $\mathrm{Cu}$ & $\left(\mathrm{mg} \mathrm{kg}^{-1} 4.17\right)$ \\
\hline High Available $\mathrm{Mn}$ & $\left(\mathrm{mg} \mathrm{kg}^{-1} 68.6\right)$ \\
\hline High Available Fe & $\left(\mathrm{mg} \mathrm{kg}^{-1} 39.4\right)$ \\
\hline
\end{tabular}

\subsection{Treatments and Experimental Design}

The experiment was laid out in a factorial Randomized Complete Block Design with three replications. Among 20 treatment $\mathrm{i}, \mathrm{e} ; \mathrm{T}_{0}=$ control, $\mathrm{T}_{1}=$ two hand weeding $(30$ days after transplanting and 45 days after transplanting), $\mathrm{T}_{2}=$ bensulfuron methyl $8 \%+$ acetachlor $14 \%, \mathrm{~T}_{3}=2$ 4, D-amine72SCL, $\mathrm{T}_{4}=$ pretilachlor 500EC, $\mathrm{T}_{5}=$ triafemon20SC, $\mathrm{T}_{6}=$ pyrazosulfuron ethyl, $\mathrm{T}_{7}=$ trisulfuron40WP,$\quad \mathrm{T}_{8}=$ pendimethalin $33 \mathrm{EC}, \quad \mathrm{T}_{9}=$ metsulfuron methyl20WDG, $\mathrm{T}_{10}=$ butachlor $3 \mathrm{G}, \mathrm{T}_{11}=$ ethoxysulfuron $150 \mathrm{WG}, \mathrm{T}_{12}=$ carfentrazol ethyl $24 \mathrm{EC}, \mathrm{T}_{13}=$ fenoxpro-p-ethyl, $\mathrm{T}_{14}=$ bensulfuron methyl20gm+bispyriback sodium $180 \mathrm{gm}, \mathrm{T}_{15}$ =penoxulam $240 \mathrm{SC}, \mathrm{T}_{16}$ $=$ pyrazosulfuron ethyl $100 \mathrm{~g}+$ pretilachlor $100 \mathrm{~g}, \mathrm{~T}_{17}=$ bispyriback sodium $300 \mathrm{WP}, \mathrm{T}_{18}=$ paraquate dichloride, $\mathrm{T}_{19}=$ glyphosate. The unit plot size was $3 \mathrm{mx} 4 \mathrm{~m}$, row and hill spacing was $(20 \times 15) \mathrm{cm}$ and test crop was Binadhan-5 HYV (duration 155 days, potential yield $9 \mathrm{t}$. ha- 1 . The treatments were randomly distributed to the plots within a block. The bunds around individual plots were sufficiently strong to control water movement between the plots. A drain of one meter wide provided for watering around the whole experimental plot and between the blocks.

\subsection{Weather Parameters}

Table 2. Weather parameters during experimental periods January to May 2019 in Mymensingh, Bangladesh.

\begin{tabular}{|c|c|c|c|c|c|c|}
\hline \multirow{2}{*}{ Month } & \multicolumn{3}{|c|}{ Temperature ${ }^{0} \mathrm{C}$} & $\begin{array}{c}\text { Rainfall } \\
(\mathrm{mm})\end{array}$ & $\mathrm{RH}(\%)$ & $\begin{array}{c}\text { Sunshine } \\
\text { hours }\end{array}$ \\
\cline { 2 - 5 } & Min. & Max. & Average & & & \\
\hline January & 19 & 29 & 24 & 2.4 & 49 & 371 \\
\hline February & 19 & 29 & 25 & 16.8 & 49 & 333 \\
\hline March & 21 & 32 & 28 & 52.5 & 48 & 372 \\
\hline April & 25 & 35 & 31 & 252.3 & 61 & 352 \\
\hline May & 26 & 36 & 32 & 413.4 & 73 & 332 \\
\hline
\end{tabular}


Vol. 06, No. 04; 2021

ISSN: $2456-8643$

\subsection{Equation}

Summed dominance ration (SDR): Summed dominance ration of weed species was computed using the following equation

Relative density (RD) + Relative dry weight (RDW)

$\mathrm{SDR}=$

2

Density of a given species

Relative density (RD) =----------------------------------- x100

Total density

Dry weight of a given species

Relative dry weight (RDW) =------------------------------- x100

Total dry weight

Weed control efficiency $($ WCE $)=\frac{\text { WC-WT }}{--------x 100}$

WC

$\mathrm{WC}=$ Average weed dry weight per unit area in weedy check

$\mathrm{WC}=$ Average weed dry weight per unit area in treated plot

Higher value of WCE indicates greater effectiveness of herbicide

Crop growth rate: Crop growth rate (CGR) expresses the increase in dry matter of a plant community on a unite area of land per unite time. The crop growth rate was calculated by the following formula

$\begin{array}{cc}1 & \mathrm{~W}_{2}-\mathrm{W}_{1} \\ \mathrm{GA} & \mathrm{T}_{2}-\mathrm{T}_{1}\end{array}$

Where, $\mathrm{W}_{1}=$ dry weight at time $\mathrm{T}_{1}$

$\mathrm{W}_{2}=$ dry weight at time $\mathrm{T}_{2}$

$\mathrm{GA}=$ ground area or land area $\left(\mathrm{m}^{2}\right)$

Relative growth rate: Relative growth rate (RGR) expresses the total plant dry weight increase in a time interval in relation to the initial weight.

$\mathrm{RGR}=\frac{\mathrm{Ln} \mathrm{W} \mathrm{W}_{2}-\mathrm{Ln} \mathrm{W}}{\mathrm{T}_{2}-\mathrm{T}_{1}}$

Where, $\mathrm{W}_{1}=$ dry weight of plant at time $\mathrm{T}_{1}$

$\mathrm{W}_{2}=$ dry weight of plant at time $\mathrm{T}_{2}$ 
Net assimilation rate: The net assimilation rate (NAR) was calculated by following formula

$$
\mathrm{NAR}=\frac{1}{\mathrm{LA}} \times \frac{\mathrm{dw}}{\mathrm{dt}} \mathrm{g} \mathrm{m}^{-2} \mathrm{day}^{-1}
$$

Where,

$\mathrm{dw}=$ dry weight increased in $\mathrm{t}$ days in $\mathrm{g}$.

$\mathrm{dt}=$ Number of days.

$\mathrm{LA}=$ Leaf area $\left(\mathrm{m}^{2}\right)$

Leaf area: The area of green leaves of sampled plants was measured from 30 DAT at 15 days interval upto 75 DAT by using automatic leaf area meter. Later leaf area index was calculated by using the following formula

Surface area of green leaves $\left(\mathrm{m}^{2}\right)$

$\mathrm{LAI}=$

Land area from where the leaves were collected $\left(\mathrm{m}^{2}\right)$

\subsection{Fertilizer application}

The plots of Boro rice were fertilized with urea $200 \mathrm{~kg} \mathrm{ha}^{-1}$, TSP $100 \mathrm{~kg} \mathrm{ha}^{-1}$, MoP $140 \mathrm{~kg} \mathrm{ha}^{-1}$, gypsum $60 \mathrm{~kg} \mathrm{ha}^{-1}, \mathrm{ZnSO}_{4} \mathrm{H}_{2} \mathrm{O}, 6 \mathrm{~kg} \mathrm{ha}^{-1}$. The whole amount at triple super phosphate, muriate of potash, gypsum and zinc sulphate (separately) were applied to the soil at the time of final land preparation. Urea was applied in three equal splits. Thirty days old seedlings were transplanting in the experimental plots.

\subsection{Herbicide application}

Treatment of different herbicide was assigned according to pre-plant, pre-emergence and postemergence basis and water level was maintained during treatment assigned. After application of pre-plant herbicide (paraquate dichloride, glyphosate) 15 days was considered to transplanting of rice seedling in timely. Treatments of herbicide application time, dose and water level maintained by according to recommendation of commercial grade pack labelled information.

\subsection{Harvesting and data collection}

The data of weed species and family were collected at 40 DAT. Dry wet of weeds for calculation of RWD, RWB and HE\%. The yield and yield contributing characters from10 random hills, the following data were recorded, plant height, number of total tillers hill ${ }^{-1}$, number of effective tillers hill ${ }^{-1}$, number of non-effective tillers hill ${ }^{-1}$, number of grain panicle ${ }^{-1}$, number of unfilled grains panicle ${ }^{-1}, 1000$ grain weight, grain yield $\left(t \mathrm{th}^{-1}\right)$, straw yield $\left(\mathrm{t} \mathrm{ha}{ }^{-1}\right)$.

\subsection{Data processing and analysis}

The data were analysed subjected to analysis of variance (ANOVA) technique and treatment mean differences were adjusted by the Multiple Comparison test and the treatment means were compared using the least significant different test (Gomez, K. A. and A. A. Gomez, 1984).

\section{RESULTS}

\subsection{Weed control efficiency $(\%)$}




\section{International Journal of Agriculture, Environment and Bioresearch}

Vol. 06, No. 04; 2021

ISSN: $2456-8643$

To calculate the weed control efficiency the weed species, weed density, weed dry weigh was recorded. The highest weed control efficiency was found at penoxsulam treated plot $86.55 \%$ lowest weed control efficiency (64.14\%) was found at 2-4, D amine72SCL (T3) treatment.

Table 3. Effect of herbicide treatment on weed species, weed density, weed dry matter and weed control efficiency

\begin{tabular}{|c|c|c|c|c|c|c|}
\hline Treatment & $\begin{array}{c}\text { Rate of } \\
\text { application }\end{array}$ & $\begin{array}{c}\text { Weed } \\
\text { Species } \\
\left(\text { no. } \mathrm{m}^{-2}\right)\end{array}$ & $\begin{array}{l}\text { Weed } \\
\text { Density } \\
\left(\text { no. } \mathrm{m}^{-2}\right)\end{array}$ & $\begin{array}{c}\text { Weed } \\
\text { Fresh wt. } \\
\left(\mathrm{g} \mathrm{m}^{-2}\right)\end{array}$ & $\begin{array}{l}\text { Weed dry } \\
\text { wt. } \\
\left(\mathrm{g} \mathrm{m}^{-2}\right)\end{array}$ & $\begin{array}{l}\text { WCE } \\
(\%)\end{array}$ \\
\hline Control $\left(\mathrm{T}_{0}\right)$ & No weeding & 17 & 37 & 124.9 & 35.7 & - \\
\hline Two hand weeding $\left(\mathrm{T}_{1}\right)$ & $\begin{array}{c}\text { Two } \\
\text { 30DAT, } \\
\text { 45DAT }\end{array}$ & 4 & 14 & 56.4 & 8.52 & 76.13 \\
\hline $\begin{array}{l}\text { Bensulfuran methyl } 8 \%+ \\
\text { Acetachlor } 14 \%\left(\mathrm{~T}_{2}\right)\end{array}$ & $180 \mathrm{~g} / \mathrm{ha}$ & 3 & 12 & 49.0 & 5.62 & 84.25 \\
\hline $2-4, \mathrm{D}$ amine $72 \mathrm{SCL}\left(\mathrm{T}_{3}\right)$ & $1.25 \mathrm{~L} / \mathrm{ha}$ & 4 & 13 & 71.4 & 12.8 & 64.14 \\
\hline Pretilachlor500EC( $\left.\mathrm{T}_{4}\right)$ & 1L/ha & 4 & 12 & 56.4 & 7.1 & 80.11 \\
\hline Triafemon $20 \mathrm{SC}\left(\mathrm{T}_{5}\right)$ & $200 \mathrm{~g} / \mathrm{ha}$ & 2 & 17 & 69.1 & 7.5 & 78.99 \\
\hline Pyrazosulfuron ethyl( $\left.\mathrm{T}_{6}\right)$ & $125 \mathrm{~g} / \mathrm{ha}$ & 4 & 11 & 50.2 & 6.7 & 81.23 \\
\hline Trisulfuron40WP( $\left.\mathrm{T}_{7}\right)$ & $400 \mathrm{~g} \mathrm{a} . \mathrm{i} / \mathrm{ha}$ & 2 & 8 & 54.0 & 7.8 & 78.15 \\
\hline Pendimethalin33EC( $\left.\mathrm{T}_{8}\right)$ & $1000 \mathrm{ml} / \mathrm{ha}$ & 4 & 13 & 71.2 & 13.3 & 62.74 \\
\hline $\begin{array}{l}\text { Metsulfuron } \\
\text { methyl20WDG(}\left(\mathrm{T}_{9}\right)\end{array}$ & $50 \mathrm{~g} / \mathrm{ha}$ & 4 & 13 & 35.4 & 8.3 & 76.75 \\
\hline Butachlor3G( $\left.\mathrm{T}_{10}\right)$ & $25 \mathrm{~kg} / \mathrm{ha}$ & 3 & 17 & 47.8 & 8.7 & 75.63 \\
\hline $\begin{array}{l}\text { Ethoxysulfuron } 150 \mathrm{WG} \\
\left(\mathrm{T}_{11}\right)\end{array}$ & $100 \mathrm{~g} / \mathrm{ha}$ & 1 & 11 & 38.9 & 6.7 & 81.23 \\
\hline $\begin{array}{l}\text { Carfentrazol ethyl } \\
24 \mathrm{EC}\left(\mathrm{T}_{12}\right)\end{array}$ & $(1.8 \mathrm{~L} / \mathrm{ha})$ & 1 & 15 & 53.3 & 8.2 & 77.03 \\
\hline
\end{tabular}




\section{International Journal of Agriculture, Environment and Bioresearch}

Vol. 06, No. 04; 2021

ISSN: $2456-8643$

\begin{tabular}{|c|c|c|c|c|c|c|}
\hline Fenoxpro-p-ethyl( $\left.\mathrm{T}_{13}\right)$ & $275 \mathrm{ml} / \mathrm{ha}$ & 3 & 13 & 67.18 & 10.5 & 70.58 \\
\hline $\begin{array}{l}\text { Bensulfuron methyl20gm } \\
+ \text { Bispyriback } \\
\text { sodium180gm( }\left(\mathrm{T}_{14}\right)\end{array}$ & $160 \mathrm{~g} / \mathrm{ha}$ & 3 & 11 & 56.38 & 6.8 & 80.95 \\
\hline Penoxsulam240SC $\left(\mathrm{T}_{15}\right)$ & $87.5 \mathrm{ml} / \mathrm{ha}$ & 4 & 15 & 56.84 & 4.8 & 86.55 \\
\hline $\begin{array}{l}\text { Pyrazosulfuran ethyl100g } \\
+ \text { Pretilachlor } 100 \mathrm{~g}\left(\mathrm{~T}_{16}\right)\end{array}$ & $200 \mathrm{~g} / \mathrm{ha}$ & 4 & 12 & 48.17 & 6.21 & 82.60 \\
\hline $\begin{array}{l}\text { Bispyriback } \\
\left.\text { sodium300WP( } \mathrm{T}_{17}\right)\end{array}$ & $200 \mathrm{~g} / \mathrm{ha}$ & 6 & 11 & 41.7 & 6.9 & 80.67 \\
\hline Paraquate dichloride $\left(\mathrm{T}_{18}\right)$ & $2.8 \mathrm{~L} / \mathrm{ha}$ & 7 & 17 & 67.8 & 15.34 & 57.03 \\
\hline Glyphosate( $\left.\mathrm{T}_{19}\right)$ & 3.7L/ha & 4 & 15 & 63.17 & 16.12 & 54.62 \\
\hline
\end{tabular}

\subsection{Particulars of weed species in the weedy check plots}

From keen observation Out of total 17 weed species annual weeds 12 species were mostly dominating and 5 weed species perennials were less dominating. Out of total 17 weed species 6 belongs to Gramineae, 1 weed species belongs to Pontederiaceae, 6 weed species belongs to Cyperaceae and 1 weed species belongs to Asteraceae, 1 weed species belongs to Marsileaceae, 1 species belongs to Sphenocleaceae family found to grow in the weedy check plots (Table 4). Morphological types were recorded that two weed species were broad-leaved, 6 were grasses and 7 weed species were sedges.

Table 4. Particulars of weed species in the weedy check plots of the experiment

\begin{tabular}{|l|l|l|l|l|l|}
\hline Sl. & Local name & Scientific name & Family & $\begin{array}{l}\text { Morphological } \\
\text { type }\end{array}$ & Life cycle \\
\hline 1 & Shama & Echinochloa colomum & Gramineae & Grass & Annual \\
\hline 2 & Gaicha & Paspalum distichum & Gramineae & Grass & Perennial \\
\hline 3 & Panikachu & Monochoria vaginalis & Pontederiaceae & Broad-leaved & Perennial \\
\hline 4 & Arail & Leersia hexandra L. & Gramineae & Grass & Annual \\
\hline 5 & Chechra & Scirpus mucronatus L. & Cyperaceae & Sedge & Perennial \\
\hline 6 & Zhilmorich & Sphenoclea zeylanica & Sphenocleaceae & Broad-leaved & Annual \\
\hline 7 & Shushnishak & Marsilea quadrifolia & Marsileaceae & Sedge & Annual \\
\hline
\end{tabular}


International Journal of Agriculture, Environment and Bioresearch

Vol. 06, No. 04; 2021

ISSN: 2456-8643

\begin{tabular}{|l|l|l|l|l|l|}
\hline 8 & Holdemutha & Cyperus difformis. & Cyperaceae & Sedge & Annual \\
\hline 9 & KhudeShama & Echinochloa colona & Gramineae & Grass & Annual \\
\hline 10 & Keshuti & Eclipta alba & Asteraceae & Sedge & Annual \\
\hline 11 & Barochucha & Cyperus iria & Cyperaceae & Sedge & Perennial \\
\hline 12 & Chechra & Scirpus maritimus & Cyperaceae & Sedge & Annual \\
\hline 13 & Durba & Cynodon dactylon & Gramineae & Grass & Perennial \\
\hline 14 & Nunia & Portulaca oleracea & Portulaceae & Broad-leaved & Annual \\
\hline 15 & Fulkaghash & Leptochloa chinensis & Gramineae & Grass & Annual \\
\hline 16 & Chotaceich & Cyperus difformis & Cyperaceae & Sedge & Annual \\
\hline 17 & Zaina & Fimbristylis miliacea & Cyperaceae & Sedge & Annual \\
\hline
\end{tabular}

\subsection{Weed ranking}

The summed dominance ratio an important pointer of showing ranking of weeds in a particular location. From close observation the dominant weed species was (Leersia hexandra. Cyperus difformis, Cyperus iria) in experiment plots.

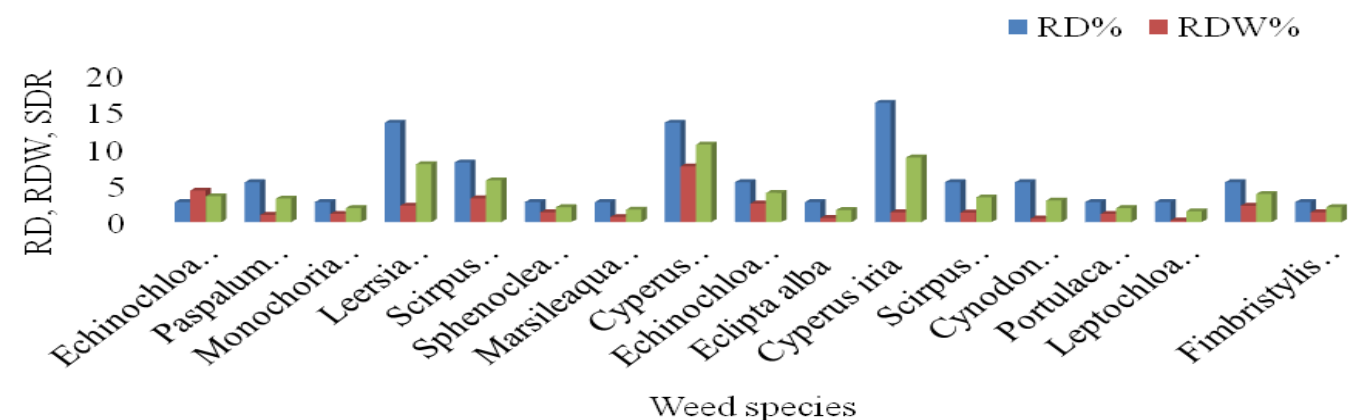

Figure 2: Weed ranking

\subsection{Weed species composition}

The maximum weed species 17 was found at control plot. Hand weeding is widely used weed control method, availability of labour in season the main limitation to its effectiveness. In Bangladesh, hand weeding rice at 30 and 45 DAT only 4 weed species was found. Minimum weed species was found in ethoxysulfuron150WG $\left(\mathrm{T}_{11}\right)$ and carfentrazol ethyl 24EC $\left(\mathrm{T}_{12}\right)$ it suppress other 16 weed species compared to treated plot (Table 5). Bensulfuran methyl 8\%+ 
Vol. 06, No. 04; 2021

ISSN: $2456-8643$

acetachlor $14 \%\left(\mathrm{~T}_{2}\right)$, butachlor $3 \mathrm{G}\left(\mathrm{T}_{10}\right)$, fenoxpro-p-ethyl $\left(\mathrm{T}_{13}\right)$, bensulfuron methyl + bispyriback sodium $\left(\mathrm{T}_{14}\right) 3$ weed species and $\left(\mathrm{T}_{3}, \mathrm{~T}_{4}, \mathrm{~T}_{6}, \mathrm{~T}_{8}, \mathrm{~T}_{9}, \mathrm{~T}_{15}, \mathrm{~T}_{16}, \mathrm{~T}_{19}\right)$ treatment 4 weed species was found (Table 5). Among the infested, different species of weeds; maximum relative weed density was observed for Echinochloa colona, Cyperus difformis, Leersia hexandra L. It was observed that broad leaf weeds were less dominating species. Due to application of preemergence or early post emergence, the herbicide gives good control over a wide range of grass, broad leaf weeds and sedges species. Single application of Pyrazosulfuron ethyl (T6) provided full control on $C$. rotundus and $F$. miliacea controlled $C$. difformis but less effective to suppress Ecliptaalba, Marsilea quadrifolia, Cyperus difformis, Cyperus iria.

Table 5. Weed species composition and effect of herbicide on weed infestation and relative weed density

\begin{tabular}{|c|c|c|c|c|}
\hline Treatment & Local name & Scientific name & $\begin{array}{c}\text { Weed } \\
\text { infestation } \\
\left(\text { no. } \mathrm{m}^{-2}\right)\end{array}$ & $\begin{array}{c}\text { Relative } \\
\text { density (\%) }\end{array}$ \\
\hline \multirow[t]{15}{*}{$\mathrm{T}_{0}$} & Shama & Echinochloa colomum & 1 & 2.70 \\
\hline & Gaicha & Paspalum distichum & 2 & 5.40 \\
\hline & Panikachu & Monochoria vaginalis & 1 & 2.70 \\
\hline & Arail & Leersia hexandra L. & 5 & 13.5 \\
\hline & Chechra & Scirpus mucronatusL. & 3 & 8.10 \\
\hline & Zhilmorich & Sphenoclea zeylanica & 1 & 2.70 \\
\hline & Shushnishak & Marsilea quadrifolia & 1 & 2.70 \\
\hline & Holdemutha & Cyperus difformis & 5 & 13.5 \\
\hline & Khudeshama & Echinochloa colona & 2 & 5.40 \\
\hline & Keshuti & Eclipta alba & 1 & 2.70 \\
\hline & Barochucha & Cyperus iria & 6 & 16.2 \\
\hline & Chechra & Scirpus maritimus & 2 & 5.40 \\
\hline & Durba & Cynodon dactylon & 2 & 5.40 \\
\hline & Nunia & Portulaca oleracea & 1 & 2.70 \\
\hline & Fulkaghash & Leptochloa chinensis & 1 & 2.70 \\
\hline
\end{tabular}


International Journal of Agriculture, Environment and Bioresearch

Vol. 06, No. 04; 2021

ISSN: $2456-8643$

\begin{tabular}{|c|c|c|c|c|}
\hline & Chotaceich & Cyperus difformis & 2 & 5.40 \\
\hline & Zaina & Fimbristylis miliacea & 1 & 2.70 \\
\hline & Total & & 37 & 100 \\
\hline \multirow[t]{5}{*}{$\mathrm{T}_{1}$} & Khudeshama & Echinochloa colona & 4 & 28.57 \\
\hline & Arail & Leersia hexandra $L$. & 2 & 14.28 \\
\hline & Holdemutha & Cyperus difformis. & 4 & 28.57 \\
\hline & Chechra & Scirpus mucronatus $L$. & 4 & 28.57 \\
\hline & Total & & 14 & 100 \\
\hline \multirow[t]{4}{*}{$\mathrm{T}_{2}$} & Shama & Echinochloa colomum & 2 & 16.66 \\
\hline & Panikachu & Monochoria vaginalis & 6 & 50.00 \\
\hline & Holdemutha & Cyperus difformis. & 4 & 33.34 \\
\hline & Total & & 12 & 100 \\
\hline \multirow[t]{5}{*}{$\mathrm{T}_{3}$} & Shama & Echinochloa colomum & 4 & 25 \\
\hline & Gaicha & Paspalum distichum & 3 & 12.5 \\
\hline & Khudeshama & Echinochloa colona & 3 & 37.5 \\
\hline & Arail & Leersia hexandra L. & 3 & 25 \\
\hline & Total & & 13 & 100 \\
\hline \multirow[t]{5}{*}{$\mathrm{T}_{4}$} & Holdemutha & Cyperus difformis. & 4 & 30.76 \\
\hline & Chechra & Scirpus mucronatus $L$. & 2 & 15.38 \\
\hline & Shushnishak & Marsilea quadrifolia & 3 & 25.00 \\
\hline & Arail & Leersia hexandra $L$. & 3 & 25.00 \\
\hline & Total & & 12 & 100 \\
\hline \multirow[t]{3}{*}{$\mathrm{T}_{5}$} & Barochucha & Cyperus iria & 4 & 23.53 \\
\hline & Gaicha & Paspalum distichum & 13 & 76.47 \\
\hline & Total & & 17 & 100 \\
\hline
\end{tabular}


International Journal of Agriculture, Environment and Bioresearch

Vol. 06, No. 04; 2021

ISSN: $2456-8643$

\begin{tabular}{|c|c|c|c|c|}
\hline \multirow[t]{5}{*}{$\mathrm{T}_{6}$} & Keshuti & Eclipta alba & 2 & 18.18 \\
\hline & Shushnishak & Marsilea quadrifolia & 5 & 45.45 \\
\hline & Holdemutha & Cyperus difformis. & 3 & 27.27 \\
\hline & Barochucha & Cyperus iria & 1 & 9.09 \\
\hline & Total & & 11 & 100 \\
\hline \multirow[t]{3}{*}{$\mathrm{T}_{7}$} & Panikachu & Monochoria vaginalis & 5 & 50 \\
\hline & Zhilmorich & Sphenoclea zeylanica & 5 & 50 \\
\hline & Total & & 10 & 100 \\
\hline \multirow[t]{5}{*}{$\mathrm{T}_{8}$} & Panikachu & Monochoria vaginalis & 1 & 7.69 \\
\hline & Arail & Leersia hexandra L. & 3 & 23.07 \\
\hline & Holdemutha & Cyperus difformis. & 5 & 38.46 \\
\hline & Chechra & Scirpus mисronatus $L$. & 4 & 30.76 \\
\hline & Total & & 13 & 100 \\
\hline \multirow[t]{5}{*}{$\mathrm{T}_{9}$} & Khudeshama & Echinochloa colona & 4 & 30.76 \\
\hline & Arail & Leersia hexandra L. & 3 & 23.07 \\
\hline & Panikachu & Monochoria vaginalis & 2 & 15.38 \\
\hline & Holdemutha & Cyperus difformis. & 4 & 30.76 \\
\hline & Total & & 13 & 100 \\
\hline \multirow[t]{4}{*}{$\mathrm{T}_{10}$} & Panikachu & Monochoria vaginalis & 4 & 23.52 \\
\hline & Zhilmorich & Sphenoclea zeylanica & 6 & 35.29 \\
\hline & Barochucha & Cyperus iria & 7 & 41.17 \\
\hline & Total & & 17 & 100 \\
\hline \multirow[t]{2}{*}{$\mathrm{T}_{11}$} & Arail & Leersia hexandra L. & 11 & 100 \\
\hline & Total & & 11 & 100 \\
\hline $\mathrm{T}_{12}$ & Khudeshama & Echinochloa colona & 14 & 100 \\
\hline
\end{tabular}


International Journal of Agriculture, Environment and Bioresearch

Vol. 06, No. 04; 2021

ISSN: $2456-8643$

\begin{tabular}{|c|c|c|c|c|}
\hline & Total & & 14 & 100 \\
\hline \multirow[t]{4}{*}{$\mathrm{T}_{13}$} & Panikachu & Monochoria vaginalis & 4 & 26.67 \\
\hline & Zhilmorich & Sphenoclea zeylanica & 5 & 33.33 \\
\hline & Barochucha & Cyperus iria & 6 & 40 \\
\hline & Total & & 15 & 100 \\
\hline \multirow[t]{4}{*}{$\mathrm{T}_{14}$} & Khudeshama & Echinochloa colona & 4 & 30.76 \\
\hline & Arail & Leersia hexandra L. & 2 & 15.38 \\
\hline & Chechra & Scirpus mисronatusL & 7 & 43.84 \\
\hline & Total & & 13 & 100 \\
\hline \multirow[t]{5}{*}{$\mathrm{T}_{15}$} & Shama & Echinochloa colomum & 1 & 12.5 \\
\hline & Keshuti & Eclipta alba & 3 & 37.5 \\
\hline & Panikachu & Monochoria vaginalis & 3 & 37.5 \\
\hline & Holdemutha & Cyperus difformis. & 1 & 12.5 \\
\hline & Total & & 8 & 100 \\
\hline \multirow[t]{5}{*}{$\mathrm{T}_{16}$} & Shama & Echinochloa colomum & 2 & 13.33 \\
\hline & Gaicha & Paspalum distichum & 8 & 53.33 \\
\hline & KhudeShama & Echinochloa colona & 3 & 20 \\
\hline & Arail & Leersia hexandra L. & 2 & 13.34 \\
\hline & Total & & 15 & 100 \\
\hline \multirow{6}{*}{$\mathrm{T}_{17}$} & Barochucha & Cyperus iria & 1 & 8.33 \\
\hline & Keshuti & Eclipta alba & 2 & 16.67 \\
\hline & Panikachu & Monochoria vaginalis & 2 & 16.67 \\
\hline & Zhilmorich & Sphenoclea zeylanica & 4 & 33.33 \\
\hline & Shama & Echinochloa colomит & 2 & 16.67 \\
\hline & Gaicha & Paspalum distichum & 1 & 8.33 \\
\hline
\end{tabular}


International Journal of Agriculture, Environment and Bioresearch

Vol. 06, No. 04; 2021

ISSN: $2456-8643$

\begin{tabular}{|c|c|c|c|c|}
\hline & Total & & 12 & 100 \\
\hline \multirow{8}{*}{$\mathrm{T}_{18}$} & Khudeshama & Echinochloa colona & 1 & 5.88 \\
\hline & Arail & Leersia hexandra L. & 3 & 17.64 \\
\hline & Chechra & Scirpus mucronatus L. & 2 & 11.76 \\
\hline & Shushnishak & Marsilea quadrifolia & 4 & 23.52 \\
\hline & Holdemutha & Cyperus difformis. & 1 & 5.88 \\
\hline & Barochucha & Cyperus iria & 3 & 17.64 \\
\hline & Keshuti & Eclipta alba & 3 & 17.64 \\
\hline & Total & & 17 & 100 \\
\hline \multirow{5}{*}{$\mathrm{T}_{19}$} & Gaicha & Paspalum distichum & 3 & 20 \\
\hline & Khudeshama & Echinochloa colona & 1 & 6.67 \\
\hline & Arail & Leersia hexandra L. & 7 & 46.67 \\
\hline & Gaicha & Paspalum distichum & 4 & 26.66 \\
\hline & Total & & 15 & 100 \\
\hline
\end{tabular}

\subsection{Effect of herbicide on crop growth rate}

Crop growth rate expresses the increase in dry matter of a community of plants on a unit area of land in a unit time. The data revealed that treatment effect of herbicide significantly influenced the crop growth rate of rice.

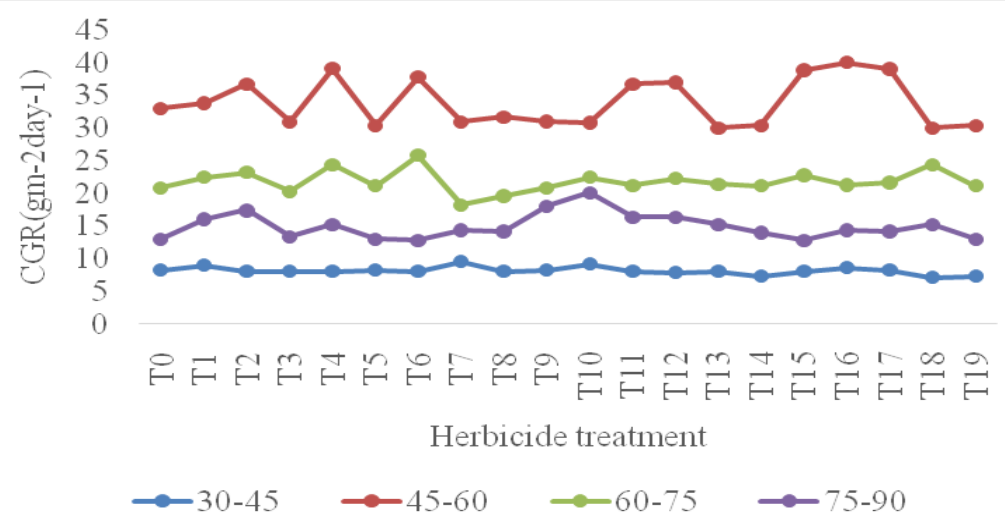

Figure 4. Effect of herbicide on net assimilation rate 
Vol. 06, No. 04; 2021

ISSN: $2456-8643$

\subsection{Effect of herbicide on relative growth rate}

Relative growth rate expresses the dry matter increase in a time interval in relation to the initial weight. The data reveals that the RGR remains same up to 30-60 DAT and thereafter, fluctuation gradually up to 60-90 DAT.

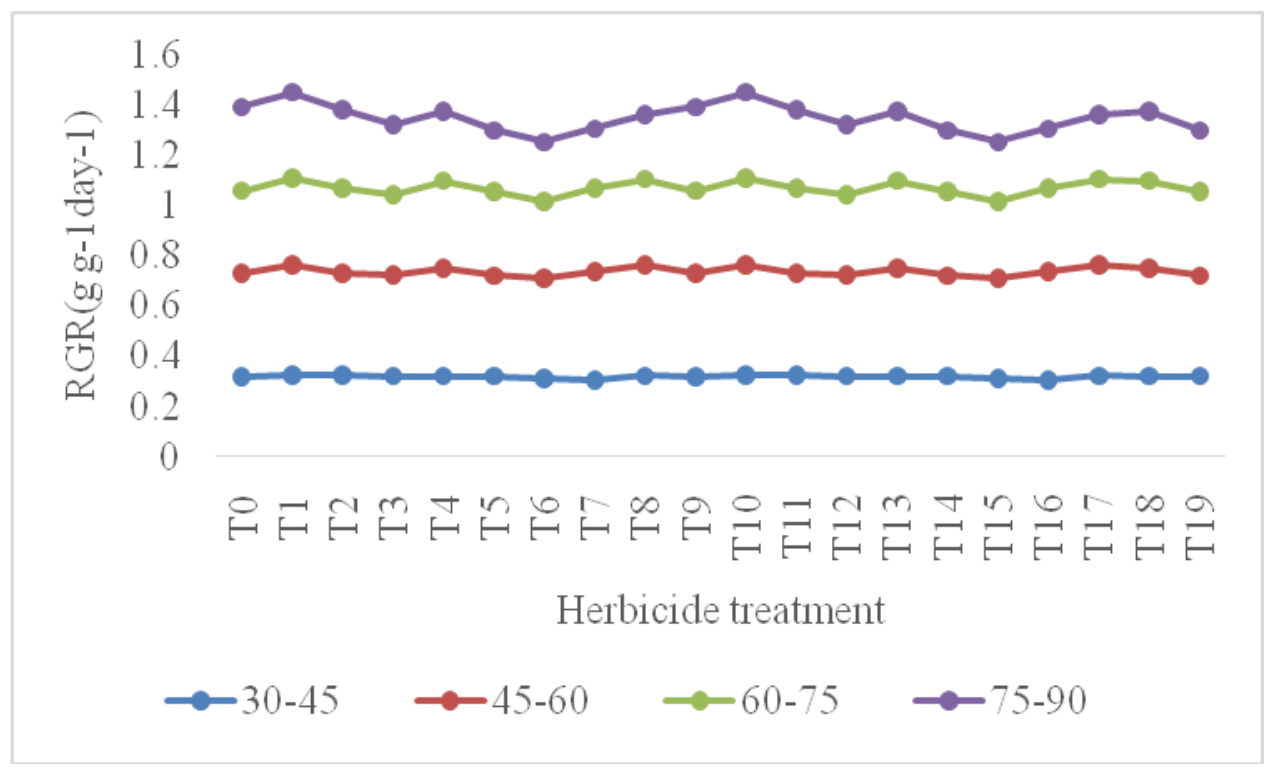

Figure 3. Effect of herbicide on net assimilation rate

\subsection{Effect of herbicide on net assimilation rate}

Net assimilation rate (NAR) is the net gain in total dry weight per unit of leaf area per unit time. The NAR is the most important index of photosynthetic efficiency of a plant. Highest net assimilation rate was found at 30 DAT and gradually it decreases significantly. Lowest NAR was found at $\mathrm{T}_{3}, \mathrm{~T}_{7}, \mathrm{~T}_{9}, \mathrm{~T}_{18}$ and $\mathrm{T}_{19}$ treatment $45 \mathrm{DAT}$.

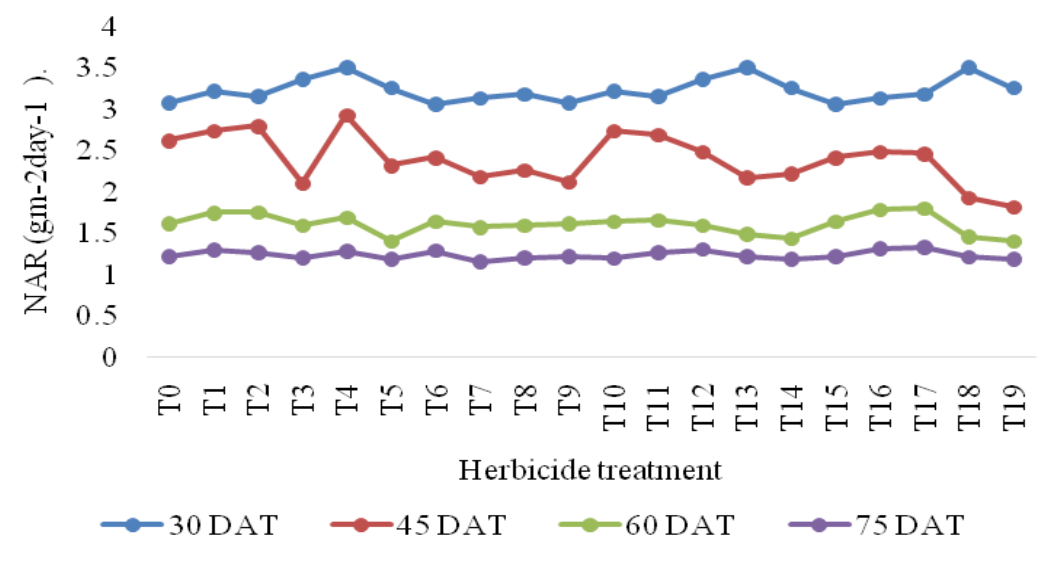

Figure 4. Effect of herbicide on net assimilation rate 
Vol. 06, No. 04; 2021

ISSN: $2456-8643$

\subsection{Effect of herbicide on yield and yield contributing characters of rice}

There is a statistically significant different in plant height and total tiller hill-1, effective tillers hill $^{-1}$, filled grains panicle ${ }^{-1}$, grain yield. Maximum plant height $(129.3 \mathrm{~cm})$ was noted for penoxulam treatment and minimum of that $(117.3 \mathrm{~cm})$ control plot. The maximum number of tillers hill ${ }^{-1}$ (10.9) was noted for penoxulam, pyrazosulfuron treatment and minimum number of tillers hill-1 (8.2) control treatment (Table 6). The maximum number of effective tillers hill-1 (8.9) was noted for penoxulam treatment and minimum number of effective tillers hill $^{-1}$ (6.3) at control treatment. The maximum filled grains panicle ${ }^{-1}$ (127.3) had obtained with application of penoxulam treatment, and minimum filled grains (104.3) was observed with control treatment.

Table 6. Effect of herbicide and weed management on yield and yield contributing characters of boro rice (Binadhan-5)

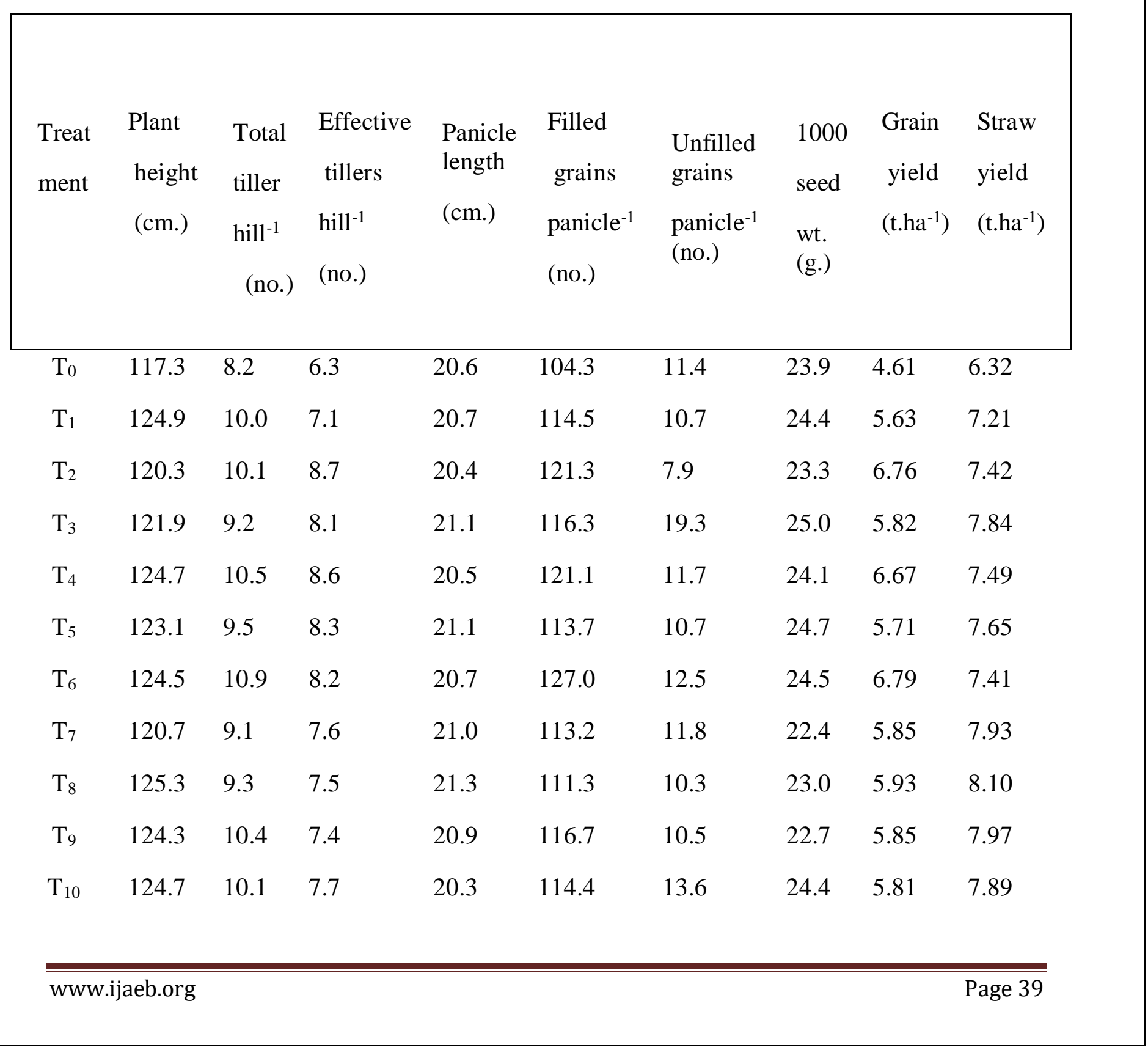


International Journal of Agriculture, Environment and Bioresearch

Vol. 06, No. 04; 2021

ISSN: $2456-8643$

\begin{tabular}{|cccccccccc}
\hline $\mathrm{T}_{11}$ & 122.7 & 10.1 & 8.1 & 21.4 & 127.1 & 11.7 & 23.0 & 6.59 & 7.33 \\
$\mathrm{~T}_{12}$ & 122.3 & 10.3 & 8.6 & 21.1 & 122.6 & 11.1 & 23.1 & 6.82 & 7.73 \\
$\mathrm{~T}_{13}$ & 123.3 & 10.3 & 7.1 & 21.0 & 111.6 & 11.6 & 23.0 & 5.87 & 7.52 \\
$\mathrm{~T}_{14}$ & 121.3 & 10.5 & 8.2 & 21.0 & 113.5 & 11.5 & 22.6 & 5.79 & 7.54 \\
$\mathrm{~T}_{15}$ & 129.3 & 10.9 & 8.9 & 21.0 & 127.3 & 17.1 & 22.6 & 6.83 & 7.60 \\
$\mathrm{~T}_{16}$ & 122.5 & 10.1 & 8.3 & 20.7 & 124.7 & 14.3 & 24.4 & 6.54 & 7.18 \\
$\mathrm{~T}_{17}$ & 125.3 & 10.3 & 8.1 & 20.5 & 123.9 & 10.7 & 24.0 & 6.61 & 7.41 \\
$\mathrm{~T}_{18}$ & 123.5 & 9.3 & 7.4 & 20.4 & 114.7 & 11.3 & 24.4 & 5.51 & 7.19 \\
$\mathrm{~T}_{19}$ & 126.3 & 10.1 & 7.8 & 20.5 & 112.9 & 11.7 & 24.0 & 5.61 & 7.42 \\
\hline $\mathrm{LSD}_{0.05}$ & 4.1 & 2.2 & 2.1 & $\mathrm{NS}$ & 6.7 & $\mathrm{NS}$ & $\mathrm{NS}$ & 0.89 & 1.13 \\
$\mathrm{CV}_{(\%)}$ & 5.5 & 7.6 & 6.9 & 3.3 & 8.4 & 7.8 & 2.4 & 7.9 & 7.2 \\
\hline
\end{tabular}

\section{DISCUSSIONS}

This chapters covers the discussion of results of following parameters; weed control efficiency

4.1 Weed control efficiency $(\%)$

From recorded data it was found that bensulfuron methyl + acetachlor, pretilachlor, pyrazosulfuron ethyl, ethoxysulfuron, bispyriback sodium, bensulfuron methyl + bispyriback sodium, penoxsulum, pyrazosulfuron ethyl + pretilachlor efficiency more than $80 \%$ (Table 3). Whereas pendimethalin 62.74 pretilachlor and pendimethalin recorded almost similar (61.59 and $61.93 \%$, respectively) value of weed control efficiency (Ghansham Payman and Surjit Singh. 2008). Al-Kothayri et al., 1990 reported that all herbicidal treatments reduced weed population significantly when compared with weedy check. It was supported by (Hasanuzzaman et al., 2008). Transplanted boro rice biomass negatively correlated with rice yield $(\mathrm{R}=--0.76918)$ (Table 3 and 6) showing high degree of association was affected due to presence of weeds which was supported by (Suresh Kumar et al., 2008).

\subsection{Weed species composition}

Hand weeding expensive task in short supply of labor in peak season and weed control found imperfect and delayed. Herbicide treatment including post-emergence herbicide was effective for broadleaf weed control which is supported by (Chauhan, B. S et al., 2015). On the contrary preemergence herbicide treatments having butachlor results less effective to suppress of sedge weeds Monochoria vaginalis, Sphenoclea zeylanica supported to the studies that butachlor was less effective on sedges in case of dry direct seeded rice (Mahajan, G. et al., 2008). Applied late post-emergence herbicide gave full control on Cyperus rotundus, Fimbristylis miliacea and $C$. difformis which is supported by (Taslima Zahan et al., 2018). Bispyriback sodium ( $\left.\mathrm{T}_{17}\right)$ treated 


\section{International Journal of Agriculture, Environment and Bioresearch}

Vol. 06, No. 04; 2021

ISSN: $2456-8643$

plot only 6 weed species found when compare with control 17 weed species, it is a selective herbicide and effective for the control of grasses, sedges and broadleaf weeds in rice which is supported by (Schmidt L A. et al., 1999). In case of trisulfuron $\left(\mathrm{T}_{7}\right)$ treated plot only two broad leaf weed species was found Monochoria vaginalis, Sphenoclea zeylanica it might be more effective to suppress grass less effective for broadleaf weed (Table 4).

\subsection{Effect of herbicide on crop growth rate (CGR)}

Among 20, the highest CGR was recorded at trisulfuron $\left(\mathrm{T}_{7}\right)$ treatment 30-45 DAT, pyrazosulfuran ethyl + pretilachlor $\left(\mathrm{T}_{16}\right)$ 45-60 DAT, pretilachlor $\left(\mathrm{T}_{4}\right)$ and Paraquate dichloride $\left(\mathrm{T}_{18}\right)$, 60-75 DAT, trisulfuron $\left(\mathrm{T}_{7}\right), 75-90$ DAT (Figure 1). It might be due to herbicide treatment initially less utilize the available nutrients in case of less microbial activity due to herbicide toxicity. After 30-45 DAT plant maximum utilize the available nutrients gradually increased leaf area, and higher photosynthesis and dry matter accumulation which enhanced crop growth rate. Herbicides have toxic effects on microorganisms, reducing their abundance, activity and consequently, the diversity of their communities. The toxic effects of herbicides are normally most severe immediately after application (Mondal et al., 2019). Afterwards microorganisms take part in a degradation process and its toxic effect gradually decline up to half-life. Then the degraded organic herbicide provides the substrate with carbon, which leads to an increase of the soil microflora.

\subsection{Effect of herbicide on relative growth rate (RGR)}

Irrespective of herbicide treatments due to leaf senescence or destruction of chlorophyll pigment and less photosynthetic activity. The highest RGR was found in the treatment with bensulfuron methyl + acetachlor $\left(\mathrm{T}_{2}\right)$, butachlor $\left(\mathrm{T}_{10}\right) \&$ fenoxpro-p-ethyl $\left(\mathrm{T}_{13}\right)$ (Figure 2$)$.

\subsection{Effect of herbicide on net assimilation rate (NAR)}

Irrespective of herbicide treatment, NAR was higher at the early growth stages and declined very sharply from 45 DAT till maturity. The highest NAR was recorded in the treatment with Pretilachlor $\left(\mathrm{T}_{4}\right)$ and Butachlor $\left(\mathrm{T}_{10}\right)$ treatment. From recorded data at later growth 75-90 DAT stages of growth with steady increase in LAI, and the lowest NAR was found (Figure 3).

\subsection{Effect of herbicide on yield and yield contributing characters of rice}

All weed control treatments significant difference on plant height, total tillers hill-1, effective tillers hill ${ }^{-1}$, more number of grains panicle ${ }^{-1}$ grain yield and straw yield and there was no significant difference on panicle length, unfilled grains panicle ${ }^{-1}, 1000$-grain weight as compared to weedy check (Ghansham Payman and Surjit Singh. 2008). The highest grain yield (6.83 tha $^{-1}$ ) was found with penoxsulam treatment which was followed by $\left(6.82 \mathrm{t} \mathrm{ha}^{-1}\right)$ at (Bensulfuran methyl + Bispyriback sodium) treatment and lowest $\left(4.61 \mathrm{t} \mathrm{ha}^{-1}\right)$ at control. It was happened due to better weed control that leads to reduced crop-weed competition and facilitates the uptake of more nutrients, proper spacing for maximum solar radiation harvest and assimilates more photosynthates that results in healthier rice plants with more tillers, panicles and biomass production to increase yield (Awan, T. H. et al., 2015 and Ahmed, S., et al., 2014). It was found that average yield varies (6.59 to 6.83) $\mathrm{t} \mathrm{ha}^{-1}$ of these chemical class bensulfuron methyl + acetachlor, pretilachlor, pyrazosulfuron ethyl, ethoxysulfuron, bispyriback sodium, bensulfuron 


\section{International Journal of Agriculture, Environment and Bioresearch}

Vol. 06, No. 04; 2021

ISSN: $2456-8643$

methyl + bispyriback sodium, penoxsulam, pyrazosulfuron ethyl + pretilachlor treated plots and their weed control efficiencies more than eighty (Table 6).

\section{CONCLUSION}

It may be concluded that grain yield maximum grain yield found with penoxsulam treatment which was followed by Bensulfuran methyl+ Bispyriback sodium treatment. From recorded data the highest weed control efficiency was found at penoxsulam which is followed by bensulfuron methyl + acetachlor and lowest glyphosate treated plot. The results suggest that for Old Brahmaputra Floodplain (AEZ-9), as like as non-calcareous dark grey flood plain soils parent material, Brahmaputra River borne deposits to maximize yield of boro rice cultivation. From efficiency point of view bensulfuron methyl + acetachlor, pretilachlor, pyrazosulfuron ethyl, ethoxysulfuron, bispyriback sodium, bensulfuron methyl + bispyriback sodium, penoxsulam, pyrazosulfuron ethyl + pretilachlor may control weed efficiently and gave maximum yield.

\section{Acknowledgements}

The authors are grateful to the Ministry of National Science and Technology Project to allocate fund conducts this experiment.

\section{REFERENCES}

Ahmed, S., Chauhan, B. S. (2014). Performance of different herbicides in dry-seeded rice in Bangladesh. The Scientific World Journal. http://dx.doi.org/10.1155/2013/729418.

Al-Kothayri, G.R. and A.A. Hasan, (1990). Chemical control of annual weeds in irrigated onion Hadhramount valley. Arab J. Plant Protec. (Lebanon), 8(1): 49-54.

Awan, T. H., Cruz, P. C. S., Chauhan, B. S. (2015). Agronomic indices, growth, yieldcontributing traits, and yield of dry-seeded rice under varying herbicides. Field Crops Resource, $177,15-25$.

Bari, M. N. (2010). Effects of herbicides on weed suppression and rice yield in transplanted wetland rice. Pakistan Journal of Weed Science Research 16, 349-361.

Chauhan B.S. (2013). Strategies to manage weedy rice in Asia. Crop Prot. 48: 51-56.

Chauhan, B. S., Awan, T. H., Abugho, S. B., Evengelista, G., Yadav, S. (2015). Effect of crop establishment methods and weed control treatments on weed management and rice yield. Field Crops Resources, 172, 72-84.

Ghansham Payman and Surjit Singh. (2008). Effect of Seed Rate, Spacing and Herbicide Use on Weed Management in Direct Seeded Upland Rice (Oryza sativa L.)Indian J. Weed Sci. 40 (1 \& 2): 12 .

Ghansham Payman and Surjit Singh. (2008). Effect of Seed Rate, Spacing and Herbicide Use on Weed Management in Direct Seeded Upland Rice (Oryza sativa L.) Indian J. Weed Sci. 40 (1 \& 2): 13 .

Ghosh RK, Mallick S and Bera S. (2013). Efficacy of bispyribac sodium 10\% SC against weed complex under different rice ecosystem. Pestology 37(9): 47-53.

Gomez, K.A. and A.A. Gomez, (1984). Statistical Procedures for Agriculture Research. Int. Rice Res. Inst. Jhon Wiley and Sons. New York, pp: 139-240.

Hasanuzzaman, M., M.O. Islam and M.S. Bapari, (2008).Efficacy of different herbicides over manual weeding in controlling weeds in transplanted rice. Aus. J. Crop Sci., 2(1): 18-24. 
Vol. 06, No. 04; 2021

ISSN: $2456-8643$

Hossain, M. M., Begum, M., Rahman, M. M., Hashem, A. (2015). Response of t. aman and boro rice to residue retention under strip tillage system. Bangladesh Agronomy Journal, 18(2), 39-44. Jabran K. and Chauhan B.S. (2015). Weed management in aerobic rice systems. Crop Prot. 78: $151-163$.

Kaloumenos N.S., Capote N., Aguado A., and Eleftherohorinos I.G. (2013). Red rice (Oryza sativa) cross-resistance to imidazolinone herbicides used in resistant rice cultivars grown in northern Greece. Pest. Biochem. Physiol. 105: 177-183.

Krishna, V., Mehrotra, M. B., Teufel, N., Bishnoi, D. K. (2012). Characterizing the cereal systems and identifying the potential of conservation agriculture in South Asia. Socio-Economics Program Working Paper 5. Mexico, D.F. CIMMYT.

Mahajan, G. Chauhan, B. S. (2008). Performance of penoxsulam for weed control in transplanted rice. Pest Technology, 2 (2), 114-116.

Mirza Hasanuzzaman, M.H. Ali, M.M. Alam, Mujahid Akther and Kazi Fakhrul Alam.(2009). Evaluation of Pre-emergence Herbicide and Hand Weeding on the Weed Control Efficiency and Performance of Transplanted Aus Rice. American-Eurasian Journal of Agronomy 2 (3): 138139.

Mondal et al., 2019. Eco-efficacy of pretilachlor 50\% EC in transplanted winter rice and its residual effect on lentil. Indian Journal of Weed Science 51(3): 225.

Najim M. M. M., Lee T.S., Haque M.A., and Esham M. (2007). Sustainability of rice production: a Malaysian perspective. J. Agric. Sci. 3: 1-12.

Schmidt L A, Talbert RE, Baldwin FL, Rutledge EFS, Wheeler SS (1999). Performance of V10029 (bispyribac) in rice weeds control programs. Proceedings South Weed Sci. Soc., 52: 49.

Siwar C., Idris N.D.M., Yasar M., and Morshed G. (2014). Issues and challenges facing rice production and food security in the granary areas in the East Coast Economic Region (ECER), Malaysia. Res. J. Appl. Sci. Eng. Technol. 7: 711-722.

Suresh Kumar et al., (2008). Efficacy of new herbicide to manage weeds in transplanted rice. Himachal Journal of Agricultural Research 34(1): 20.

Taslima Zahan et al., (2018). Efficacy of herbicides in non-puddled transplanted rice under conservation agriculture systems and their effect on establishment of the succeeding crops. Acta Scientifica Malaysia (ASM) 2(1):17-25.

Zeliger, H. I., Human toxicology of chemical mixtures (2011). In: Toxic Consequences beyond the Impact of One-component Product and Environmental Exposures. 2nd ed. Elsevier, Oxford. 